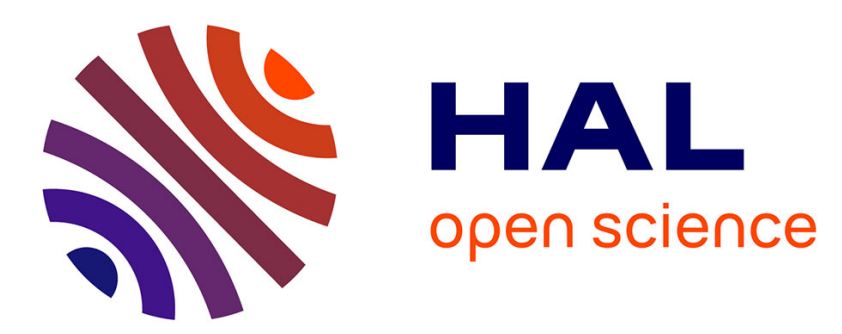

\title{
Introducing Consistency in a Dialogical Framework for Paraconsistent Logic (online 2018)
}

Eduardo Barrio, Nicolas Clerbout, Shahid Rahman

\section{To cite this version:}

Eduardo Barrio, Nicolas Clerbout, Shahid Rahman. Introducing Consistency in a Dialogical Framework for Paraconsistent Logic (online 2018). Logic Journal of the IGPL, 2020, 28 (5), pp.953-972. halshs-01689148

\section{HAL Id: halshs-01689148 \\ https://shs.hal.science/halshs-01689148}

Submitted on 21 Jan 2018

HAL is a multi-disciplinary open access archive for the deposit and dissemination of scientific research documents, whether they are published or not. The documents may come from teaching and research institutions in France or abroad, or from public or private research centers.
L'archive ouverte pluridisciplinaire HAL, est destinée au dépôt et à la diffusion de documents scientifiques de niveau recherche, publiés ou non, émanant des établissements d'enseignement et de recherche français ou étrangers, des laboratoires publics ou privés. 


\title{
Introducing Consistency in a Dialogical Framework for Paraconsistent Logic
}

\author{
Eduardo Barrio • Nicolas Clerbout • \\ Shahid Rahman
}

Received: date / Accepted: date

\begin{abstract}
The Logics of Formal Inconsistency are logics tolerant to some amount of inconsistency, but in which some versions of explosion still hold. The main result of this paper is a reconstruction of two such logics in the dialogical framework. By doing so, we achieve two things. On the one hand, we provide a formal approach to argumentative situations where some inconsistencies may occur while keeping the idea that there may still be situations in which some propositions are "safe" in the sense of immunity to the contradictions. On the other hand, we open a new line of study on these logics, in the context of the game-theoretical approach to semantics born in the 1960s, with various interesting perspectives, some of which are discussed at the end of this article.
\end{abstract}

\section{Introduction}

In the context of paraconsistent systems, white bullets or circles (o) have been introduced in order to develop logics that, though tolerant to (some) contradictions, rest as near as possible to classical logic. These logics are known

Eduardo Barrio

IIF-Conicet

University of Buenos Aires

E-mail: eabarrio@gmail.com

Nicolas Clerbout

Instituto de Filosofía - CDHACS, University of Valparaíso

Conicyt; Fondecyt Iniciación 11160397

E-mail: nicolas.clerbout@uv.cl

Shahid Rahman

Univ. Lille, CNRS, UMR 8163 - STL

F-59000 Lille, France

E-mail: shahid.rahman@univ-lille3.fr 
as Logics of Formal Inconsistency (LFIs). In a nutshell, LFIs are systems for which there are some $\Gamma, A, B$ such that: ${ }^{1}$

$-\Gamma, A, \neg A \not \models B$

$-\Gamma, A, \circ A \not \models B$

$-\Gamma, \circ A, \neg A \not \models B$

And yet for all $\Gamma, A, B$ :

$-\Gamma, \circ A, A, \neg A \vDash B$

Along these lines, $\circ$ is usually interpreted as a consistency operator, and $\circ A$ means that $A$ is consistent. ${ }^{2}$ So, the consistency of a proposition is expressed in the object-language by using the aforementioned logical operator. Those properties show that despite the fact that in LFIs explosion does not in general hold, some versions of it do hold when the atomic propositions at stake are assumed to be consistent. Indeed, $\circ A$ may express the consistency independent from freedom from contradiction. ${ }^{3}$ Originally ${ }^{4}$ this notion was a primitive that cannot be semantically characterized by finite matrices. Da Costa's System mbC is an example of this limitation, but some paraconsistent logics with consistency operator can be defined by a matrix providing a many-valued semantics for this operator. For example, Halldén's logic of nonsense as well as Segerberg's variation, da Costa and D'Ottaviano's logic J3 (also known in its variants LFI1 and MPT), Sette's logic P1, the system Ciore, and several other related systems.

The aim of this paper is to provide this kind of operator with a dialogical meaning that should also naturally lead to tableaux systems. In fact, it seems that the result applies to any paraconsistent logic, though we will focus on the logics P1 (Sette) and MPT (Coniglio, M. \& Da Cruz Silvestrini, L., 2014,): the former is a logic that has already been studied in a dialogical framework (Rahman \& Carnielli 2000) and MPT can be seen as resulting from a kind of hierarchy based on the level of generalization of the indexes attached to bottom. ${ }^{5}$

\footnotetext{
1 As usual, $\Gamma$ is a set of formulas and $A, B$ are formulas.

2 We want to be neutral about the different philosophical interpretations that the operator o can receive. Maybe contradictions are connected with some problems about information or reality. But the point of introducing and studying the operator $\circ$ is to have a way to distinguish between contradictions that can be accepted from those that cannot. The point of this distinction is that no matter the nature of the contradictions a paraconsistentist is willing to accept, there are contradictions that cannot be accepted. Because of that, the logicians working in the LFIs tradition think of their logical frameworks as recovering classical reasoning when working under the assumption that some of the sentences in question are consistent in the sense that contradictions on them are not acceptable. See Carnielli and Coniglio. (2016, p. 17). We would like to thank one of the anonymous referees for drawing attention to this point.

3 cf. Carnielli, W., Coniglio, M, \& Marcos, J. 2007 and Carnielli, W., and Coniglio, M. 2016).

4 cf. Da Costa. N. 1963, 1993 and Da Costa, N. 1974

5 We are going to focus on the logics P1 and MPT because these systems have received comparatively less attention than Halldén and Segerberg's logics and J3 in the framework
} 
The paper will be developed in four steps. We first introduce two different variations on the standard rules for dialogical games, thus giving two different examples of how paraconsistency can be introduced in dialogue games of argumentation: in the first case, the tolerance for inconsistencies is very restricted and actually limited to the use of atomic formulas, while in the second case elementary inconsistencies may very well affect complex formulas. In the second step, we present a way to introduce a certain amount of consistency in an otherwise paraconsistent dialogue game by means of a special operator ensuring that certain sequences of the game must be played without allowing inconsistencies. After that, we make explicit the relationship between the two dialogical systems presented and two particular LFIs, Sette's logic P1 on the one hand and the propositional logic of pragmatic truth MPT on the other hand. The article ends with some general concluding remarks and perspectives for future investigation on the potential of the dialogical approach to LFIs.

\section{The dialogical version of $\mathrm{P} 1$ and of the circle-free fragment of MPT}

The dialogical framework born in the 1950s from the work of Lorenzen and Lorenz offers an alternative paradigm on logic in which meaning is defined in terms of interaction between a Proponent $(\mathbf{P})$ and an Opponent $(\mathbf{O})$ within an argumentative game. Accordingly, the dialogical approach is a pragmatist theory of meaning based on the well-known dictum "meaning is use". However, here "use" is to be understood as "use as prescribed by a rule of dialogical interaction", rather than as a set of inference rules. In that way, the meaning of expressions is internal to that space of rational argumentation we call a dialogical play.

Meaning is defined by two kinds of rules. Particle rules determine the local meaning in terms of assertions, requests, challenges, and defences. Structural rules give the general conditions of the game and shape the global level of meaning. The standard particle rules are:

\begin{tabular}{|c|c|c|c|c|}
\hline Assertion & $\mathbf{X} ! A \wedge B$ & $\mathbf{X} ! A \vee B$ & $\mathbf{X} ! A \supset B$ & $\mathbf{X} ! \neg A$ \\
\hline Challenge & $\mathbf{Y} ?_{L}$ or $\mathbf{Y} ?_{R}$ & $\mathbf{Y} ?_{\vee}$ & $\mathbf{Y} ! A$ & $\mathbf{Y} ! A$ \\
\hline Defence & $\mathbf{X} ! A$ or $\mathbf{X} ! B$ & $\mathbf{X} ! A$ or $\mathbf{X} ! B$ & $\mathbf{X} ! B$ & -- \\
\hline
\end{tabular}

The standard structural rules for classical games are the following: SR0 (Starting Rule): Let $A$ be a complex formula. Any play for $A$ starts with $\mathbf{P}$ asserting $A$. Then $\mathbf{O}$ and $\mathbf{P}$ successively choose a positive integer. $A$ is called the thesis of the play (and the game), and the positive integers are called repetition ranks of the players.

of traditional model theory. P1 and MPT have interesting philosophical applications: P1 is motivated by intuition according to which the paraconsistency only happens on the atomic level: any complex formula is 'classical' in the sense that it can only take the truth-values 0 or 1 . MPT can be seen as a paraconsistent logic that analyses the notion of Quasi-Truth in partial structures (or pragmatic structures). 
SR1 (Classical Game-Playing Rule): After the choices of repetition ranks, players make their moves in turns. Let $n$ be player $\mathbf{X}$ 's repetition rank. When he has a turn to play, $\mathbf{X}$ can challenge a previous assertion of $\mathbf{Y}$ or defend against a previous challenge by $\mathbf{Y}$, accordingly to the particle rules and as long as he has already responded to the relevant move of $\mathbf{Y}$ less than $n$ times. SR2 (Formal Rule): $\mathbf{P}$ can assert an atomic formula only if $\mathbf{O}$ has already asserted it before.

The Formal Rule makes sure that the Proponent (when defending the thesis against the Opponent's challenges) is not freely bringing in elementary statements in order to back his assertions. On the other hand, using the very elementary statements of the Opponent in order to back the thesis ensures that the justification is kept internally in the dialogue and rests on concessions of the adversary.

SR3 (Winning Rule): Player $\mathbf{X}$ wins a play only if he played the last move in the current play and there is no allowed move for $\mathbf{Y}$ to extend the play.

According to SR3, winning amounts to having the last word in the debate. The Proponent winning means that he successfully defended his thesis against the attacks performed by the Opponent. On the contrary, the Opponent winning means that his criticisms were efficient. It is important to notice that the notion of winning is always relative to a play, and that means that it is relative to particular ways of playing from the players. For another level of analysis, we need the notion of strategies, as explained below.

The standard structural rules for intuitionistic games are the same, except that SR1 is replaced by the following intuitionistic version:

SR1i (Intuitionistic Game-Playing Rule): After the choices of repetition ranks, players make their moves in turns. Let $n$ be player $\mathbf{X}$ 's repetition rank. When he has a turn to play, $\mathbf{X}$ can challenge a previous assertion of $\mathbf{Y}$, provided he has challenged it less than $n$ times already or defend against the last unanswered challenge by $\mathbf{Y}$.

The difference between SR1 and SR1i can be explained as follows. In SR1i, the repetition rank keeps applying to challenges while restrictions on defences are much stronger than in SR1: an adversary challenge can be answered at most one time, but provided there is no previous unanswered adversary challenge. This illustrates one of the virtues of the dialogical framework that allows switching smoothly between systems to provide semantics for different logics at stake. Our aim is to take advantage of this in order to offer a dialogical perspective on the paraconsistent logic $\mathbf{P} \mathbf{1}$ and the circle-free fragment of the logic MPT. We will deal with a dialogical version of the circle operator in a subsequent section.

Before that, we need to recall one important aspect of the dialogical approach to logic, which is the existence of another level of analysis in addition to the play-level governed by the rules. This is the level of strategies. A strategy for player $\mathbf{X}$ in a given dialogical game is simply a function that selects a legal move for $\mathbf{X}$ to play whenever it is his turn to play, provided there is at least such a move. When such a strategy allows $\mathbf{X}$ to win no matter how the 
adversary $\mathbf{Y}$ plays, then the strategy is said to be winning. The existence of a winning strategy for $\mathbf{P}$ in a given dialogical system of rule is the dialogical notion of validity for this system. For example, we described the two sets of rules above as rules for respectively "classical" and "intuitionistic" games: this is because the existence of a winning strategy for $\mathbf{P}$ in the game for a given formula $A$ corresponds to the validity of $A$ in classical (respectively, intuitionistic) logic. Demonstrations of these correspondences can be found in other works. ${ }^{6}$

2.1 Dealing with negative literals: a first dialogical approach to paraconsistency

We can now move to the general topic of paraconsistency in the dialogical framework, before dealing with the particular cases of P1 and MPT. Let us start then by recalling some of the ideas outlined in the seminal paper (Rahman/Carnielli, 2000) on the dialogical approach to paraconsistency. The first idea is to read the negation in terms of material implication and bottom, and to distinguish between various occurrences of a bottom with indexes. That is, to work with $A \supset \perp$ for $\neg A$. The point of the index is to distinguish between various occurrences of the bottom, so that on the one hand players do not necessarily lose by asserting any bottom and on the other hand it is not the case that any occurrence of bottom allows to conclude an arbitrary formula meaning that the rule explosion is not generally valid. For example, the index ${ }^{7}$ allows distinguishing between $A \supset \perp_{A}$ and $B \supset \perp_{B}$.

In addition to the use of indexed bottoms for the negation, the systems introduced in Rahman/Carnielli (2000) feature a modified version of the formal rule SR2 in which not only positive atomic formulas are considered but also negative literals - that is to say, negations of atomic formulas:

SR2 $_{P 1}$ (Formal Rule for atomic formulas and negative literals): $\mathbf{P}$ can assert an atomic formula only if $\mathbf{O}$ has already asserted it before. In the case where the atomic formula $p$ is to be asserted in order to challenge the negative literal $p \supset \perp_{p}, \mathbf{P}$ can perform the challenge only if $\mathbf{O}$ has already challenged $p \supset \perp_{p}$ previously.

With these modifications of the standard dialogical framework, Rahman and Carnielli offer a new understanding of the semantic assumptions of paraconsistent logics by giving ways to distinguish between various kinds of contradictions (depending on the index on the bottom, or whether a player is being contradictory with the adversary or with himself, for example). Let us

\footnotetext{
6 For example, Rahman(1993), Keiff(2007) or Clerbout(2014c).

7 The motivation for the indexation of bottom can be seen as a natural extension of the rationale behind Johansson's minimal logic. In minimal logic the introduction of of bottom always occurs in the context of an implication, more precisely as the consequent of the implication. The idea of indexes is to keep track of the formula occurring as the antecedent of the implication.
} 
consider an example with a play for the formula

$$
(a \supset b) \supset\left(\left((a \supset b) \supset \perp_{1}\right) \supset \perp_{2}\right)
$$

Example 1

\begin{tabular}{|c|c|c|c|c|c|}
\hline & $\mathbf{O}$ & & & $\mathbf{P}$ & \\
\hline & & & & $!(a \supset b) \supset\left(\left((a \supset b) \supset \perp_{1}\right) \supset \perp_{2}\right)$ & 0 \\
\hline 1 & $\mathrm{n}:=1$ & & & $\mathrm{~m}:=2$ & 2 \\
\hline 3 & $! a \supset b$ & $(0)$ & & $!\left((a \supset b) \supset \perp_{1}\right) \supset \perp_{2}$ & 4 \\
\hline 5 & $!(a \supset b) \supset \perp_{1}$ & $(4)$ & & $! a \supset b$ & \\
\hline & & & $(5)$ & $! b$ & 10 \\
\hline 7 & $! a$ & $(6)$ & & $! a$ & 8 \\
\hline 9 & $! b$ & & $(3)$ & \multicolumn{2}{c}{} \\
\hline
\end{tabular}

Such a table provides a convenient representation of a play, insofar as it keeps track of the order of the moves (by means of the numbers in the outer columns, starting with 0 for the thesis) while showing which moves are challenged (by means of the integers in the inner columns) and which moves are answers to challenges - a defence is written in front of the corresponding challenge.

In this example, the game proceeds as follows: after the thesis has been asserted and the repetition ranks chosen, the Opponent challenges the Proponents moves according to the particle rule for material implication up until move 5. The Proponent must counterattack with move 6 because he cannot assert $\perp_{2}$ to respond to move 5 . Next the Opponent counterattacks with move 7. The play ends after the Proponent forces the sequence of moves 8 to 10 , using what is called copy-cat moves: he mirrors the Opponent's moves. After move 10 the Opponent does not have any possible move, so the play is won by the Proponent.

From what we explained above, merely winning a play is in general not enough to conclude that $\mathbf{P}$ has a winning strategy - i.e. that he can win no matter how the Opponent plays. However, we can rely on some results and notions introduced and explained in previous works to conclude in this case that the Proponent does have a winning strategy in this game. ${ }^{8}$ These results, to summarize it, ensure us that the problem of the existence of winning strategies can be simplified by looking at what is called the cores of strategies. A core of a strategy is basically a collection of plays in a given game, obeying certain conditions which make them the relevant plays to consider in order to determine whether a given strategy is winning or not. An important aspect of this line is the possibility to ignore most of the possible repetition ranks for the players and limit the verification to the case where the Opponent's rank is 1 and the Proponent's is 2. Unless specified otherwise, all the examples in the current study are such plays constituting cores of strategies and in this respect allow (through the previous results we have just mentioned) to conclude about the existence or non-existence of a winning strategy for the Proponent.

8 See in particular Clerbout(2014a,b) and Clerbout \& Rahman(2015). 
For a second example let us consider an instance of explosion. ${ }^{9}$

Example 2

\begin{tabular}{|c|c|c|c|c|c|}
\hline & $\mathbf{O}$ & & & $\mathbf{P}$ & \\
\hline & & & & $\left(a \wedge\left(a \supset \perp_{1}\right)\right) \supset b$ & 0 \\
\hline 1 & $\mathrm{n}:=1$ & & & $\mathrm{~m}:=2$ & 2 \\
\hline 3 & $a \wedge\left(a \supset \perp_{1}\right)$ & $(0)$ & & & \\
\hline 5 & $a$ & & $(3)$ & $?_{L}$ & 4 \\
\hline 7 & $a \supset \perp_{1}$ & & $(3)$ & $?_{R}$ & 6 \\
\hline
\end{tabular}

In this play, the Proponent cannot answer to the Opponent's move 3 because he cannot assert $b$. This is why he counterattacks with moves 4 and 6 . However, there is nothing he can do after that. In particular, he cannot attack move 7 because of rule SR2 $2_{P 1}$. The idea is that $a$ is not an explosive formula in the sense that the fact that $\mathbf{O}$ asserted it and its negation is not enough to allow $\mathbf{P}$ to assert $b$. The Opponent wins this play, and that shows that there is not a winning strategy for $\mathbf{P}$ in the game for this instance of Explosion.

Let us call $D_{P 1}$ the set of dialogical rules which consists of the standard classical rules with $\mathrm{SR} 2_{P 1}$ instead of SR2. The set $D_{P 1 i}$ is $D_{P 1}$ with SR1i instead of SR1. In Section 4 we will show that $D_{P 1}$ captures Sette's logic P1 in the sense that the existence of a winning strategy for $\mathbf{P}$ in a $D_{P 1}$ game corresponds to $\mathbf{P} \mathbf{1}$ provability. To see that this is not the case with $D_{P 1 i}$, it is enough to exhibit a $\mathbf{P 1}$ provable formula for which there is no winning strategy for $\mathbf{P}$ in the $D_{P 1 i}$ game. Let us consider $\left(\left(a \supset \perp_{1}\right) \supset \perp_{2}\right) \supset a$. We know from Proposition 4 in Sette (1973) that it is a theorem of P1. Nevertheless, the following dialogue shows that there is no winning strategy for $\mathbf{P}$ under the rules of $D_{P 1 i}$.

\section{Example 3}

\begin{tabular}{|c|c|c|c|c|c|}
\hline & $\mathbf{O}$ & & & $\mathbf{P}$ & \\
\hline & & & & $\left(\left(a \supset \perp_{1}\right) \supset \perp_{2}\right) \supset a$ & 0 \\
\hline 1 & $\mathrm{n}:=1$ & & & $\mathrm{~m}:=2$ & 2 \\
\hline 3 & $\left(a \supset \perp_{1}\right) \supset \perp_{2}$ & $(0)$ & & & \\
\hline & & & $(3)$ & $a \supset \perp_{1}$ & 4 \\
\hline 5 & $a$ & $(4)$ & & & \\
\hline
\end{tabular}

The play proceeds with challenges and counterattacks in accordance with the particle rules. The Opponent does assert $a$ at move 5, which is precisely the atomic formula $\mathbf{P}$ needs to be able to defend against $\mathbf{O}$ 's challenge 3 . However, the structural rule SR1i forbids him to do so because move 5 is an unanswered challenge, so that move 3 is no longer the last unanswered challenge by $\mathbf{O}$. There is nothing the Proponent can do after move 5, so he loses this play. Moreover, there is nothing he can do to prevent this development, therefore there is not a winning strategy for him in this game.

Let us now move to the circle-free fragment of the paraconsistent logic MPT.

9 From now on, we will omit the exclamation marks when writing assertions in the examples. 
2.2 Transmissible non explosive absurdities: an example of a more fine-grained approach to paraconsistency

An alternative formulation of SR2 $P 1$ is that, although asserting an absurdity is in general prohibited (or equivalently, a reason to lose the play), the Opponent can assert an elementary absurdity $\perp_{a}$ if $\mathbf{P}$ challenged the negative literal $a \supset \perp_{a}$ without her challenging it beforehand. The underlying idea is that elementary absurdities are not always explosive. Thus we can read SR2 $2_{P 1}$ as introducing some level of paraconsistency in the use of atomic formulas. Even so, it is obviously possible to consider other rules introducing other forms of paraconsistency. In this section, we give one example.

Let $D_{\perp}$ be the set of rules consisting of the standard classical rules but with the following rule $\mathrm{SR} 2_{\perp}$ instead of SR2:

$\mathrm{SR2}_{\perp}($ Formal Rule for atomic formulas and $\perp$ ):

1. Let $p$ be an atomic formula other than an absurdity $\perp_{X}$ (where $X$ is any formula). $\mathbf{P}$ can assert $p$ only if $\mathbf{O}$ has already asserted it before

2. (General case for absurdities) If $\mathbf{O}$ asserted $\perp_{X}$ beforehand, then $\mathbf{P}$ can assert $\perp_{X}$

3. (Special cases for absurdities): Let $A$ and $B$ be any formulas:

(a) Let $C$ be any formula other than an absurdity $\perp_{X}$. A player asserting $\perp_{(A \supset C)}$ loses the play.

(b) If $\mathbf{O}$ asserted $A, \perp_{A}$ and $B$, then $\mathbf{P}$ can assert $\perp_{(A \wedge B)}$.

(c) If $\mathbf{O}$ asserted $A$ and $\perp_{A}$, then $\mathbf{P}$ can assert $\perp_{\left(A \supset \perp_{A}\right)}$

(d) If $\mathbf{X}$ asserted $\left(A \supset \perp_{A}\right) \supset \perp_{\left(A \supset \perp_{A}\right)}$, then $\mathbf{X}$ cannot assert $\perp_{\left(A \supset \perp_{A}\right)}$

While $\mathrm{SR} 2_{P 1}$ introduces paraconsistency only at the level of atomic formulas, this other rule is more liberal in the sense that it allows (under specific conditions) the assertion of absurdities including for some complex formulas. Notice that this rule to some extent is nothing but one possibility where paraconsistency can be transmitted from the atomic level to negations and conjunctions (and disjunctions too, as a result), but not to implications. ${ }^{10}$ Insofar as absurdities are atomic statements even when they stem from complex formulas, the conditions for their use are to be given by the Formal Rule. Of course, there are other possibilities to be explored, with rules more or less liberal than $\mathrm{SR} 2_{\perp}$, but for the current work we will consider the cases of negations and conjunctions, and leave implications aside for the time being.

The first point of SR $\perp_{\perp}$ states that the atomic formulas (other than absurdities) behave like in classical dialogues: the Proponent can assert them only if the Opponent asserted them previously. The second and third points deal with absurdities and distinguish between the general case where $\mathbf{P}$ is allowed to assert an absurdity and special conditions for allowing or prohibiting the

\footnotetext{
10 One way to understand this idea of transmission of paraconsistency in some cases but not in the case of implication is to think of how some formulas can and cannot get the third value in three-valued semantics for LFIs, in this case for MPT.
} 
assertion of some kinds of absurdity. Item 3(a) states that absurdities stemming from a usual material implication are explosive. Item 3(b) states that non-explosive absurdity can be "transmitted" through conjunction under certain conditions. Item $3(\mathrm{c})$ is similar to the case of negation, but item $3(\mathrm{~d})$ puts a restriction on the iterative use of $3(\mathrm{c})$.

\section{Example 4}

\begin{tabular}{|c|c|c|c|c|c|}
\hline & $\mathbf{O}$ & & & $\mathbf{P}$ & \\
\hline & & & & $a \supset\left(\left(a \supset \perp_{a}\right) \supset \perp_{\left(a \supset \perp_{a}\right)}\right)$ & 0 \\
\hline 1 & $\mathrm{n}:=1$ & & & $\mathrm{~m}:=2$ & 2 \\
\hline 3 & $a$ & $(0)$ & & $\left(a \supset \perp_{a}\right) \supset \perp_{\left(a \supset \perp_{a}\right)}$ & 4 \\
\hline 5 & $a \supset \perp_{a}$ & $(4)$ & & $\perp_{\left(a \supset \perp_{a}\right)}$ & 8 \\
\hline 7 & $\perp_{a}$ & & $(5)$ & $a$ & 6 \\
\hline
\end{tabular}

The Proponent's victory in this play results from a direct application of item $3(\mathrm{c})$ of rule $\mathrm{SR} 2_{\perp}$ in order for him to play move 8.

\section{Example 5}

\begin{tabular}{|c|c|c|c|c|c|}
\hline & $\mathbf{O}$ & & & $\mathbf{P}$ & \\
\hline & & & & $\left(\left(a \wedge\left(a \supset \perp_{a}\right)\right) \wedge b\right) \supset\left(\left((a \wedge b) \supset \perp_{(a \wedge b)} \wedge\left((b \wedge a) \supset \perp_{(b \wedge a)}\right)\right.\right.$ & 0 \\
\hline 1 & $\mathrm{n}:=1$ & & & $\left((a \wedge b) \supset \perp_{(a \wedge b)}\right) \wedge\left((b \wedge a) \supset \perp_{(b \wedge a)}\right)$ & 2 \\
\hline 3 & $\left(a \wedge\left(a \supset \perp_{a}\right)\right) \wedge b$ & $(0)$ & & $(a \wedge b) \supset \perp_{(a \wedge b)}$ & 4 \\
\hline 5 & $?_{L}$ & $(4)$ & & $\perp_{(a \wedge b)}$ & 6 \\
\hline 7 & $a \wedge b$ & $(6)$ & & $?_{L}$ & 18 \\
\hline 9 & $a \wedge\left(a \supset \perp_{a}\right)$ & & $(3)$ & $?_{R}$ & 10 \\
\hline 11 & $b$ & & $(3)$ & $?_{L}$ & 12 \\
\hline 13 & $a$ & & $(9)$ & $?_{R}$ & 14 \\
\hline 15 & $a \supset \perp_{a}$ & & $(9)$ & $a$ & 16 \\
\hline 17 & $\perp_{a}$ & & $(13)$ & $(9)$ & 8 \\
\hline
\end{tabular}

Here, the Proponent wins by applying item $3(\mathrm{~b})$ of SR $2_{\perp}$. Indeed he is able to force the Opponent to assert successively $b, a$ and $\perp_{a}$. This allows him to assert $\perp_{a \wedge b}$ and win the play.

\section{The circle operator in the dialogical framework}

In this section, we provide the dialogical approach to the full-fledged paraconsistent logic MPT by introducing a dialogical equivalent to the circle operator o.

As we have seen above, one way to understand the o operator at an intuitive level is that it introduces limited consistency in an otherwise paraconsistent system. In dialogical terminology, this operator is understood as having a structural effect (in the sense of pertaining to the level of structural rules) in addition to having a local behavior in terms of challenge and defence.

Operators of this kind have been studied in some works in the dialogical tradition, and we will take inspiration from one in particular in order to define a dialogical operator capturing the o operator of MPT. This system is an LFI that can be formulated as follows: 
Definition 1 Let the language $\mathcal{L}_{\mathrm{MPT}}$ be composed of a denumerable set of propositional variables $\vee$ ar and a set $C_{\mathbf{M P T}}=\left\{\sim, \neg, \wedge, \vee, \circ, \supset, \leftrightarrow_{\mathbf{M P T}}\right\}$ of connectives. The set of formulae Form $\mathcal{L}_{\text {MPт }}$ is defined, standardly, as the absolutely free algebra generated by Var over $\mathrm{C}_{\mathrm{MPT}}$.

It is worth noticing that $\leftrightarrow_{\mathrm{MPT}}$ is just the conjunction of the left-right and the right-left conditionals, as in classical logic.

Definition 2 The logic MPT $=\left\langle\mathcal{L}, \models_{\text {MPT }}\right\rangle$ is defined by the semantic structure $\mathcal{M}_{\mathrm{MPT}}=\left\langle\mathcal{V}_{\mathrm{MPT}}, \mathcal{D}_{\mathrm{MPT}}, \mathcal{O}_{\mathrm{MPT}}\right\rangle$, built in the following way:

$-\mathcal{V}_{\mathrm{MPT}}=\left\{1, \frac{1}{2}, 0\right\}$

$-\mathcal{D}_{\mathrm{MPT}}=\left\{1, \frac{1}{2}\right\}$

- $\mathcal{O}_{\mathrm{MPT}}=$ the set of truth-functions associated with the connectives in

$\mathrm{C}_{\mathrm{MPT}}$, displayed in the truth-tables of Figure 1

\begin{tabular}{c|c}
$\neg A$ & $A$ \\
\hline 0 & 1 \\
$\frac{1}{2}$ & $\frac{1}{2}$ \\
1 & 0
\end{tabular}
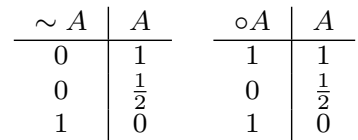

\begin{tabular}{c|ccc}
$A \wedge B$ & 1 & $\frac{1}{2}$ & 0 \\
\hline 1 & 1 & $\frac{1}{2}$ & 0 \\
$\frac{1}{2}$ & $\frac{1}{2}$ & $\frac{1}{2}$ & 0 \\
0 & 0 & 0 & 0
\end{tabular}

\begin{tabular}{c|ccc}
$A \vee B$ & 1 & $\frac{1}{2}$ & 0 \\
\hline 1 & 1 & 1 & 1 \\
$\frac{1}{2}$ & 1 & $\frac{1}{2}$ & $\frac{1}{2}$ \\
0 & 1 & $\frac{1}{2}$ & 0
\end{tabular}

\begin{tabular}{c|ccc}
$A \supset B$ & 1 & $\frac{1}{2}$ & 0 \\
\hline 1 & 1 & 1 & 0 \\
$\frac{1}{2}$ & 1 & 1 & 0 \\
0 & 1 & 1 & 1
\end{tabular}

\begin{tabular}{c|ccc}
$A \leftrightarrow_{\text {MPT }} B$ & 1 & $\frac{1}{2}$ & 0 \\
\hline 1 & 1 & 1 & 0 \\
$\frac{1}{2}$ & 1 & 1 & 0 \\
0 & 0 & 0 & 1
\end{tabular}

Fig. 1 Truth-tables for the logic MPT

The following calculus LPT (the logic of pragmatic truth) coincides with MPT, that is, LPT is sound and complete w.r.t. the matrix of MPT (Carnielli, W. and Coniglio, M. 2016, Theorem 4.4.49). Silvestrini (2011) introduced LPT as the basic paraconsistent logic relative to quasi-truth theories. The same set of axioms is presented by Coniglio \& Silvestrini (2013). And an equivalent system, but with some different axioms is introduced by (Carnielli, W. and Coniglio, M. 2016).

\section{System LPT: Axiom schemas}

(A1) $\alpha \supset(\beta \supset \alpha)$

(A2) $(\alpha \supset \beta) \supset((\alpha \supset(\beta \supset \gamma)) \supset(\alpha \supset \gamma))$

(A3) $\alpha \supset(\beta \supset(\alpha \wedge \beta))$

(A4) $(\alpha \wedge \beta) \supset \alpha$

(A5) $(\alpha \wedge \beta) \supset \beta$

(A6) $\alpha \rightarrow(\alpha \vee \beta)$

(A7) $\beta \supset(\alpha \vee \beta)$

$(\mathrm{A} 8)(\alpha \supset \gamma) \supset((\beta \supset \gamma) \supset((\alpha \vee \beta) \supset \gamma))$

(A9) $\alpha \vee(\alpha \supset \beta)$

(A10) $\alpha \vee \neg \alpha$ 


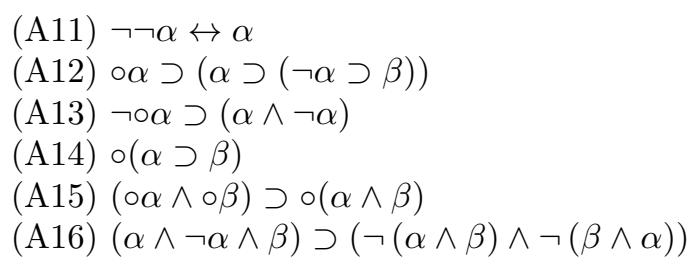

Rule of inference: (MP) infer $\beta$ from $\alpha$ and $\alpha \supset \beta$.

Observe that (A1)-(A9) plus MP constitute a Hilbert axiomatic calculus for positive classical propositional logic: the negation-free fragment of classical logic which is, in fact, the basis for minimal logic $\mathbf{m b C}$ and its extensions as LPT). The Axioms (A1) and (A2) plus the rule MP assure that the Deduction Theorem holds for LPT. The Axiom (A12) is equivalent to the formula $(\alpha \wedge$ $\neg \alpha \wedge \circ \alpha) \supset \beta$. This appears that if $\{\alpha, \neg \alpha, \circ \alpha\} \subseteq \Gamma$, then for every formula $\beta$ we have $\Gamma \vdash \beta$. This axiom is called the gentle explosion law and it is the characteristic axiom of the system mbC. The Axiom (A13) is also important for the paraconsistent character of the LPT, because this axiom shows that a formula and its negation can occur in certain situations. Sometimes (A13) is called (ci). Observe that (A1)-(A13) plus MP constitute the system mbCci. In this logic the schema (cc) $\circ \circ \alpha$ and the schema (ciw) $\circ \alpha \supset(\alpha \wedge \neg \alpha)$ are derivable. Given a class of consistent formulas, an important issue is to understand how this consistency propagates towards simpler or more complex formulas. The axiom (A14) is called (cv3). This axiom warrants that material implications are always consistent in MPT. One important property of the system MPT is the following: what we have is that paraconsistency is transmissible from the atoms to complex formulas in the cases of conjunction, disjunction, and negation, but not through material implication. Finally, the axiom (16) is called (ca1). This axiom reflects directly on the propagation of consistency through conjunctions.

\subsection{The dialogical operator $\mathbb{V}$}

Rahman and Rückert (2001) introduced and studied the dialogical approach to connexive logic. Among other things, the dialogical system presented for this purpose features operators having the kind of effect we just discussed. For the scope of the present work, we will focus on the operator $\mathbb{V}$.

Let us consider the standard dialogical rules for classical logic as presented in Section 2 - that is to say, with the standard formal rule SR2 - and extend the language with the $\mathbb{V}$ operator such that if $A$ is a formula so is $\mathbb{V} A$. The idea is that when an assertion of $\mathbb{V} A$ is challenged and defended, a certain section is opened in the play - we will call it a sub-play — and a particular change in the structural rules of the game may occur. To make this idea more precise, let us recall the actual particle rule from Rahman and Rückert (2001) for the $\mathbb{V}$ operator. 


\begin{tabular}{|c|c|}
\hline Assertion & $\mathbf{X} ! \mathbb{V} A$ \\
\hline Challenge & $\mathbf{Y} ? \mathbb{V}$ \\
\hline Defence & $\mathbf{X} ! A$ \\
& $\begin{array}{c}\text { The defender chooses the sub-play } \\
\end{array}$ \\
& The challenger must play formally in the sub-play \\
\hline
\end{tabular}

The second condition, about the challenger playing formally in the subplay, is the one on that we are interested in. In a dialogical game, playing formally means being subject to the formal rule and that is to say having a constraint on the use of atomic formulas. In the standard rules, the one playing under this constraint is the Proponent. Now the point is that the particle rule for $\mathbb{V}$ introduces the possibility for that to change in the course of a play: typically, a play starts with the standard rules and $\mathbf{P}$ playing formally, but if at some point $\mathbf{O}$ challenges a $\mathbf{P}$-assertion $\mathbb{V} A$, the formal constraint will shift and the Opponent is the one who will have to play formally.

The dialogical operator $\mathbb{V}$ is thus an operator effecting at the structural level of the course of the game, opening a sub-play in which the formal constraint coming from the rule SR2 can change. Incidentally, this is precisely why we are interested in this particular operator for our current study. Indeed it is at the structural level of rules that paraconsistency is introduced in the dialogical systems we consider, as we have seen in Section 2. This is why the operator $\mathbb{V}$ is a promising starting point to formulate a dialogical approach to the consistency operator o of MPT.

\subsection{Introducing the operator $\mathbb{V}^{\circ}$}

Regardless, we cannot simply use only the $\mathrm{V}$ operator to provide a dialogical reading of the consistency operator $\circ$. We need a different solution - a specific one - in order to provide a dialogical approach to MPT.

Paraconsistency in the dialogical systems we have introduced so far is implemented at the level of the formal rule, which governs the use of elementary formulas. More precisely, the focus is on the use of indexed absurdities of the form $\perp_{A}$. It is therefore also at this level that we need to take action in order to be able to introduce limited consistency by means of a dialogical operator emulating the intended interpretation of the MPT operator $\circ$. In this sense since the dialogical operator $\mathrm{V}$ produces certain modifications on the formal rule SR2 it is an interesting starting point.

But the modifications on SR2 triggered by the operator $\mathbb{V}$ are limited to changing the player which has to play under the formal restriction. They do not operate on the consistent or paraconsistent features of the dialogical system. More than a transfer of the formal restriction to another player, we need an operator which produces a deeper change in the rules of the game, basically allowing a paraconsistent version of SR2 to be replaced (within certain limits) by the classical version. In addition, it is worth insisting on the fact that such a change must not be limited to a player and should apply to the general conditions of the game instead. 
From these considerations, the idea is to work with an operator $\mathbb{V}^{\circ}$ which will have such a structural effect. The exact scope of this effect is, in turn, to be specified in the formulation of the formal rule. The particle rule of our new operator is the following:

\begin{tabular}{|c|c|}
\hline Assertion & $\mathbf{X} ! \mathbb{V}^{\circ} A$ \\
\hline Challenge & $\mathbf{Y} ? \mathbb{V}^{\circ}$ \\
\hline Defence & $\mathbf{X} ! A$ \\
\hline
\end{tabular}

As for the structural effect of the operator, the idea is that when $\mathbf{X}$ defends, what we call a consistent sequence is opened in the dialogue. We need to specify in the structural rules how exactly the opening of these sequences is regulated. This is done by means of the following new version of SR2:

SR2 $\mathbb{V}^{\circ}$ (Formal Rule for atomic formulas, $\perp$ and $\mathbb{V}^{\circ}$ ):

1. The game starts with $\mathrm{SR} 2_{\perp}$ as the active formal rule for atomic formulas and absurdities.

2. Whenever a player asserts $A$ in order to defend an assertion $\mathbb{V}^{\circ} A$, this opens a consistent sequence for $A$ :

(a) Any move resulting from a finite chain of applications of rules starting with $A$ belongs to the consistent sequence for $A$

(b) For any move in the consistent sequence for $A$, the active formal rule is the classical SR2 rule: asserting absurdities is prohibited, and $\mathbf{P}$ can assert an atomic formula in the consistent sequence only if $\mathbf{O}$ asserted it beforehand in a consistent sequence

(c) In a consistent sequence, $\mathbf{O}$ can challenge an implication $A \supset B$ only if $B$ is an absurdity $\perp_{X}$

3. Interactions between consistent and paraconsistent sequences:

(a) If $\mathbf{X}$ asserted $A$ in a consistent sequence, then $\mathbf{X}$ cannot assert $\perp_{A}$

(b) If $\mathbf{O}$ asserted $\left(\mathbb{V}^{\circ} A \supset \perp_{(\mathbb{V} \circ A)}\right)$, then $\mathbf{P}$ is allowed to assert $\perp_{A}$ and, for any atomic formula $a$ in $A, \mathbf{P}$ is allowed to assert $a$ and also $\perp_{a}$

Item 2(b) of the rule ensures that once an atomic formula has been granted as consistent by the Opponent, the Proponent can always use it in subsequent consistent sequences. Indeed, the idea is that once consistency has been introduced for a formula, it does not change and turn inconsistent again afterwards. Item 2(c) ensures that material implications which are not negations are always accepted as consistent. This is a complement to the fact that, according to $\mathrm{SR} 2_{\perp}$, paraconsistency is not transmitted to material implications. Just as was the case with $\mathrm{SR} 2_{\perp}$, there are other possible versions of SR $2_{\mathbb{V}}$ and of item 2 (c) in particular. In fact, it is not surprising: when a variation is introduced on how paraconsistency is managed by variations on $\mathrm{SR} 2_{\perp}$, it is natural for it to have consequences on how consistent sequences are played. For example if we imagine a system in which paraconsistency spreads through material implication but not through conjunction, item 2(c) would probably be about conjunctions and not implications. 
Item 3(a) ensures that rule $\mathrm{SR} 2_{\perp}$ cannot be wrongly used by $\mathbf{X}$ to introduce absurdities resulting from formulas that he himself played in consistent sequences. Item $3(\mathrm{~b})$ deals with the case where $\mathbf{O}$ negates that a formula behaves classically, ensuring that the Proponent can use it in a paraconsistent way.

We let $D_{M P T}$ be the set of rules with the particle rule for $\mathbb{V}^{\circ}$ and $\mathrm{SR} 2_{\mathbb{V}}$ instead of SR2. We now give some examples of plays in $D_{M P T}$

Example 6

\begin{tabular}{|c|c|c|c|c|c|}
\hline & $\mathbf{O}$ & & & $\mathbf{P}$ & \\
\hline & & & & $\left(\mathbb{V}^{\circ} a \wedge \mathbb{V}^{\circ} b\right) \supset \mathbb{V}^{\circ}(a \wedge b)$ & 0 \\
\hline 1 & $\mathrm{n}:=1$ & & & $\mathrm{~m}:=2$ & 2 \\
\hline 3 & $\mathbb{V}^{\circ} a \wedge \mathbb{V}^{\circ} b$ & $(0)$ & & $\mathbb{V}^{\circ}(a \wedge b)$ & 12 \\
\hline 5 & $\mathbb{V}^{\circ} a$ & & $(3)$ & $?_{L}$ & 4 \\
\hline 7 & $a$ & & $(5)$ & $?_{\mathbb{V}^{\circ}}$ & 6 \\
\hline 9 & $\mathbb{V}^{\circ} b$ & & $(3)$ & $?_{R}$ & 8 \\
\hline 11 & $b$ & & $(9)$ & $?_{\mathbb{V}}$ & 10 \\
\hline 13 & $?_{\mathbb{V}^{\circ}}$ & $(12)$ & & $a \wedge b$ & 14 \\
\hline 15 & $?_{L}$ & $(14)$ & & $a$ & 16 \\
\hline
\end{tabular}

Example 7

\begin{tabular}{|c|c|c|c|c|c|}
\hline & $\mathbf{O}$ & & & $\mathbf{P}$ & \\
\hline & & & & $(a \wedge b) \supset \mathbb{V}^{\circ}(a \wedge b)$ & 0 \\
\hline 1 & $\mathrm{n}:=1$ & & & $\mathrm{~m}:=2$ & 2 \\
\hline 3 & $a \wedge b$ & $(0)$ & & $\mathbb{V}^{\circ}(a \wedge b)$ & 4 \\
\hline 5 & $?_{\mathbb{V}^{\circ}}$ & $(2)$ & & $a \wedge b$ & 6 \\
\hline 7 & $?_{L}$ & $(6)$ & & & \\
\hline 9 & $a$ & & $(3)$ & $?_{L}$ & 8 \\
\hline
\end{tabular}

In Example 6 we have an illustration of how item 2(b) of the rule works: after $\mathbf{O}$ asserted that $a$ is consistent with moves 5 and $7, \mathbf{P}$ is allowed to play $a$ in the subsequent consistent sequence for $a \wedge b$ and win the game. Things are different in Example 7: the Opponent's assertion of $a$ in move 9 is not made within a consistent sequence, and for that reason the Proponent cannot use it to answer the Opponent's challenge of move 7.

Example 8

\begin{tabular}{|c|c|l|c|c|c|}
\hline & $\mathbf{O}$ & & & $\mathbf{P}$ & \\
\hline & & & & $\left(\mathbb{V}^{\circ} a \supset \perp_{\mathbb{V}^{\circ} a}\right) \supset\left(a \wedge\left(a \supset \perp_{a}\right)\right)$ & 0 \\
\hline 1 & $\mathrm{n}:=1$ & & & $\mathrm{~m}:=2$ & 2 \\
\hline 3 & $\mathbb{V}^{\circ} a \supset \perp_{\mathbb{V}^{\circ} a}$ & $(0)$ & $a \wedge\left(a \supset \perp_{a}\right)$ & 4 \\
\hline 5 & $?_{R}$ & $(4)$ & $a \supset \perp_{a}$ & 6 \\
\hline 7 & $a$ & $(6)$ & $\perp_{a}$ & 8 \\
\hline
\end{tabular}


This last example shows a simple application of item $3(\mathrm{~b})$ in rule $\mathrm{SR} 2 \mathbb{V}$. By asserting $\mathbb{V}^{\circ} a \supset \perp_{\mathbb{V}^{\circ} a}$, the Opponent grants that $a$ is not to be played classically. That is to say, it is not to be used in a consistent sequence. This allows the Proponent to play $\perp_{a}$ in move 8 , in accordance with item $3(\mathrm{~b})$, and win the play.

\section{Correspondence results}

\section{1 $D_{P 1}$ captures the logic P1}

Theorem 1 If a formula $A$ is $\mathbf{P 1}$ provable, then there is a winning strategy for $\mathbf{P}$ in the $D_{P 1}$ game for $A$.

Proof In order to prove this, it is enough to show that there is a winning strategy for each axiom of $\mathbf{P 1}$ and that the existence of winning strategies for $\mathbf{P}$ in $D_{P 1}$ is closed under Modus Ponens. As for the axioms, in what follows we present in each case a play which is representative and allows to conclude that there is a winning strategy in the following way: these are won plays which are straightforwardly extended to winning strategies because the only variations are in the order of the moves and possible repetitions. Using terminology from Clerbout (2014), they constitute cores of winning strategies. See also our remark in Section 2.1 about repetition ranks and footnote 4 .

We consider the axioms in Sette (1973), with the negation read in terms of implication and bottoms.

- Axiom 1: $A \supset(B \supset A)$
\begin{tabular}{|c|c|c|c|c|c|}
\hline & $\mathbf{O}$ & & & $\mathbf{P}$ & \\
\hline & & & & $A \supset(B \supset A)$ & 0 \\
\hline 1 & $\mathrm{n}:=1$ & & & $\mathrm{~m}:=2$ & 2 \\
\hline 3 & $A$ & $(0)$ & & $B \supset A$ & 4 \\
\hline 5 & $B$ & $(4)$ & & $A$ & 6 \\
\hline
\end{tabular}

This play is won by the Proponent after move 6 . The general idea on which rests any winning strategy for $\mathbf{P}$ is that $\mathbf{O}$ asserts at move 3 the very formula $\mathbf{P}$ needs in order to win. Notice that if $A$ is a complex formula the play will go on but the Proponent can always win by copy-cat.

- Axiom 2: $(A \supset(B \supset C)) \supset((A \supset B) \supset(A \supset C))$
\begin{tabular}{|c|c|c|c|c|c|}
\hline & $\mathbf{O}$ & & & $\mathbf{P}$ & \\
\hline & & & & $(A \supset(B \supset C)) \supset((A \supset B) \supset(A \supset C))$ & 0 \\
\hline 1 & $\mathrm{n}:=1$ & & & $\mathrm{~m}:=2$ & 2 \\
\hline 3 & $A \supset(B \supset C)$ & $(0)$ & & $(A \supset B) \supset(A \supset C)$ & 4 \\
\hline 5 & $A \supset B$ & $(4)$ & & $A \supset C$ & 6 \\
\hline 7 & $A$ & $(6)$ & & $C$ & 14 \\
\hline 9 & $B \supset C$ & & $(3)$ & $A$ & 10 \\
\hline 11 & $B$ & & $(5)$ & $A$ & 12 \\
\hline 13 & $C$ & & $(9)$ & $B$ & \\
\hline
\end{tabular}


In this play, the Proponent uses moves 8, 10 and 12 to make the Opponent assert the formula which he needs in order to respond to challenge 7. Again, if any of $A, B$ or $C$ is a complex formula and the Opponent attacks it when $\mathbf{P}$ asserts it, he can launch copy-cat moves to successfully defend his assertions.

- Axiom 3:

$$
\left(\left(A \supset \perp_{1}\right) \supset\left(B \supset \perp_{2}\right)\right) \supset\left(\left(\left(A \supset \perp_{1}\right) \supset\left(\left(B \supset \perp_{2}\right) \supset \perp_{3}\right)\right) \supset A\right)
$$

\begin{tabular}{|c|c|c|c|c|c|}
\hline & $\mathbf{O}$ & & & $\mathbf{P}$ & \\
\hline & & & & $\left(\left(A \supset \perp_{1}\right) \supset\left(B \supset \perp_{2}\right)\right) \supset\left(\left(\left(A \supset \perp_{1}\right) \supset\left(\left(B \supset \perp_{2}\right) \supset \perp_{3}\right)\right) \supset A\right)$ & 0 \\
\hline 1 & $\mathrm{n}:=1$ & & & $\mathrm{~m}:=2$ & 2 \\
\hline 3 & $\left(A \supset \perp_{1}\right) \supset\left(B \supset \perp_{2}\right)$ & $(0)$ & & $\left(\left(A \supset \perp_{1}\right) \supset\left(\left(B \supset \perp_{2}\right) \supset \perp_{3}\right)\right) \supset A$ & 4 \\
\hline 5 & $\left(A \supset \perp_{1}\right) \supset\left(\left(B \supset \perp_{2}\right) \supset \perp_{3}\right)$ & 4 & & $A$ & 14 \\
\hline 7 & $B \supset \perp_{2}$ & & (3) & $A \supset \perp_{1}$ & 6 \\
\hline 9 & $\left(B \supset \perp_{2}\right) \supset \perp_{3}$ & & $(5)$ & $A \supset \perp_{1}$ & 8 \\
\hline & & & $(9)$ & $B \supset \perp_{2}$ & 10 \\
\hline 11 & $B$ & (10) & & & \\
\hline & & & (7) & $B$ & 12 \\
\hline 13 & $A$ & (6) & & & \\
\hline 15 & $A$ & (8) & & & \\
\hline & & & & $A$ & 16 \\
\hline
\end{tabular}

- Axiom 4: $\left(\left(A \supset\left(\left(A \supset \perp_{1}\right) \supset \perp_{2}\right)\right) \supset \perp_{3}\right) \supset A$
\begin{tabular}{|c|c|c|c|c|c|}
\hline & $\mathbf{O}$ & & & $\left(\left(A \supset\left(\left(A \supset \perp_{1}\right) \supset \perp_{2}\right)\right) \supset \perp_{3}\right) \supset A$ & 0 \\
\hline & & & & $\mathrm{m}:=2$ & 2 \\
\hline 1 & $\mathrm{n}:=1$ & $(0)$ & & $A$ & 6 \\
\hline 3 & $\left(A \supset\left(\left(A \supset \perp_{1}\right) \supset \perp_{2}\right)\right) \supset \perp_{3}$ & & $(3)$ & $A \supset\left(\left(A \supset \perp_{1}\right) \supset \perp_{2}\right)$ & 4 \\
\hline & & $(4)$ & & & \\
\hline 5 & $A$ &
\end{tabular}

- Axiom 5: see example 1 in Section 1.

- Closure under Modus Ponens. Let us suppose that there is a winning strategy for $\mathbf{P}$ in the $D_{P 1}$ games for $A$ and for $A \supset B$. Now there are three general ways $\mathbf{P}$ can have a winning strategy in the game for $A \supset B$ : (i) by challenging the antecedent when asserted by $\mathbf{O}$ so that $\mathbf{O}$ cannot defend it; (ii) by defending the consequent using concessions which the Opponent has to make in order to defend the antecedent; (iii) and by defending the consequent without needing such concessions. ${ }^{11}$

In our case, there cannot be a winning strategy for $\mathbf{P}$ in the game for $A \supset B$ resting on the impossibility for $\mathbf{O}$ to defend the antecedent. This is because if there is a way for $\mathbf{P}$ to win against the $\mathbf{O}$-assertion of $A$, then surely there is a way for $\mathbf{O}$ to win against the $\mathbf{P}$-assertion of $A$. However, in that case, there is no winning strategy for $\mathbf{P}$ in the $D_{P 1}$ game for $A$, which contradicts one of our assumptions.

11 To understand how these three general ways work, the reader can think about the following examples: $\left((a \supset b) \wedge\left((a \supset b) \supset \perp_{1}\right)\right) \supset c ;(a \wedge b) \supset(b \wedge a)$; and $c \supset(a \supset(b \supset a))$. In the first case $\mathbf{P}$ wins by challenging the antecedent until $\mathbf{O}$ cannot defend anymore. In the second case $\mathbf{P}$ can win only by copying the moves of the Opponent and use the very concessions she makes when defending the antecedent. Finally, in the last case, $\mathbf{P}$ can defend the consequent without needing the antecedent. 
Let us then suppose that $\mathbf{P}$ cannot defend $B$ without using the concessions $\mathbf{O}$ has to make in order to defend $A$. This actually means that in order to defend $B$ the Proponent needs to assert some atomic formulas $a_{1}, a_{2}, \ldots$ asserted by $\mathbf{O}$ in her defence of $A$. Yet, in this case (and going back to the game for $A$ ), $\mathbf{P}$ also needs to assert the atomic formulas $a_{1}, a_{2}, \ldots$ in order to defend it. The Opponent is necessarily the one who introduces these atomic formulas because of the formal rule $\mathrm{SR} 2_{P 1}$. Going back to the game for $A, \mathbf{P}$ also needs to assert the atomic formulas $a_{1}, a_{2}, \ldots$ Regardless, the formal rule prevents him from being able to introduce them. Thus, once again, there would be no winning strategy for $\mathbf{P}$ in the game for $A$ and the original assumption would be contradicted.

In conclusion, the only possibility is that a winning strategy for the Proponent in the game for $A \supset B$ consists in successfully defending $B$ independently of the antecedent, and this amounts to $\mathbf{P}$ having a winning strategy in the $D_{P 1}$ game for $B$.

Theorem 2 If there is a winning $\mathbf{P}$ strategy in the $D_{P 1}$ game for a formula $A$, then $A$ is $\mathbf{P} \mathbf{1}$ provable

Proof We start by supposing that there is a winning $\mathbf{P}$ strategy in the $D_{P 1}$ game for $A$. For each of the atomic formulas $a_{i}$ occurring in $A$, we define $\overline{a_{i}}$ and $a_{i}^{\prime}$ as follows, ${ }^{12}$ based on assertions by the players in the strategy.

(i) If $a_{i}$ is asserted by $\mathbf{O}$ without her asserting $a_{i} \supset \perp_{1}$ and without $\mathbf{P}$ asserting $a_{i}$, we let

$$
\begin{aligned}
& \bar{a}_{i}=a_{i} \supset\left(\left(a_{i} \supset \perp_{1}\right) \supset \perp_{2}\right) \\
& a_{i}^{\prime}=a_{i}
\end{aligned}
$$

(ii) If $a_{i}$ is asserted by $\mathbf{P}$, we let

$$
\begin{aligned}
& \bar{a}_{i}=a_{i} \supset\left(\left(a_{i} \supset \perp_{1}\right) \supset \perp_{2}\right) \\
& a_{i}^{\prime}=a_{i} \supset \perp_{1}
\end{aligned}
$$

(iii) If $a_{i}$ and $a_{i} \supset \perp_{1}$ are both asserted by $\mathbf{O}$, without $\mathbf{P}$ challenging $a_{i} \supset \perp_{1}$, we let

$$
\begin{aligned}
& \bar{a}_{i}=\left(a_{i} \supset\left(\left(a_{i} \supset \perp_{1}\right) \supset \perp_{2}\right)\right) \supset \perp_{3} \\
& a_{i}^{\prime}=a_{i}
\end{aligned}
$$

It can then be shown by induction that

$$
\overline{a_{1}}, \ldots, \overline{a_{n}}, a_{1}^{\prime}, \ldots, a_{n}^{\prime} \vdash_{\mathbf{P} 1} A
$$

(See Lemma 2 in Sette(1973), with each of the cases (i)-(iii) above corresponding to the cases listed in Sette's Lemma). Now every $a_{i}^{\prime}$ can be successively eliminated in the following way:

\footnotetext{
12 This is inspired by the methodology followed by Sette in his 1973 paper to prove the completeness result (Proposition 9 and Lemma 2 which is a step in the proof of completeness).
} 
(a) If $a_{i}^{\prime}=a_{i}$, then we have

$$
\overline{a_{1}}, \ldots, \overline{a_{n}}, a_{1}^{\prime}, \ldots, a_{n-1}^{\prime} \vdash_{\mathbf{P 1}} a_{i} \supset A
$$

(b) If $a_{i}^{\prime}=a_{i} \supset \perp_{1}$, then we have

$$
\overline{a_{1}}, \ldots, \overline{a_{n}}, a_{1}^{\prime}, \ldots, a_{n-1}^{\prime} \vdash_{\mathbf{P 1}}\left(a_{i} \supset \perp_{1}\right) \supset A
$$

(c) Since $\vdash_{\mathbf{P 1}}(A \supset B) \supset(((A \supset \perp) \supset B) \supset B)$ (Sette's Proposition 6), it follows from (a) and (b) that

$$
\overline{a_{1}}, \ldots, \overline{a_{n}}, a_{1}^{\prime}, \ldots, a_{n-1}^{\prime} \vdash_{\mathbf{P 1}} \supset A
$$

A similar technique can be used for the $\overline{a_{i}} \mathrm{~s}$, so that after a finite number of steps we end with $\vdash_{\text {P1 }} A$.

Discussion. With the fact that $D_{P 1}$ captures $\mathbf{P} 1$ proven, there are some interesting remarks that can now be made. First, it is well-known that $\mathbf{P 1}$ is paraconsistent only at the atomic level, and $D_{P 1}$ gives a quite natural gametheoretical account of it: the rule SR2 $2_{P 1}$ puts restrictions on the assertions by $\mathbf{P}$ of atomic formulas, and it is the additional restriction on negative literals that allows the Opponent to introduce paraconsistent atomic formulas as formulas which she can both assert and negate without being challenged by the Proponent. In the dialogical system $D_{P 1}$, paraconsistency ends with complex formulas because the restriction for the Proponent does not prevent the Opponent to be challenged if she contradicts herself on a complex formula.

An interesting feature of $D_{P 1}$ is that it is not directly a three-valued system: the rules of the game are still formulated using a unique form of assertion (as opposed, for example, to Rückert's multivalued dialogical systems in his 2004 work). That is to say, paraconsistency is not introduced as a specific additional value for formulas. However, as illustrated by the proof of completeness we have just seen, the structural rule SR2 ${ }_{P 1}$ can be understood as allowing to distinguish between various kinds of atomic formulas according to how they are used by the players.

\section{2 $D_{M P T}$ captures the logic MPT}

Theorem 3 If a formula $A$ is LPT provable, then there is a winning strategy for $\mathbf{P}$ in the $D_{M P T}$ game for $A$.

Proof The proof is similar to what we have done in the case of $\mathbf{P 1}$ : it is easy to check that there is a winning $\mathbf{P}$ strategy for the axioms of LPT - in fact we have considered axioms (A11), (A16), (A15) and (A13) in Examples 4, 5, 6 and 8 respectively - and the closure under Modus Ponens is guaranteed just as with $\mathbf{P 1}$.

Theorem 4 If there is a winning strategy for $\mathbf{P}$ in the $D_{M P T}$ game for $A$, then $A$ is LPT provable 
Proof The clearest and simplest proof is an indirect one. The idea is to show how to interpret the players' assertions in the game for $A$ regarding the threevalued matrices of MPT. The dialogical game for $A$ can then be interpreted as the attempt by the Opponent to build a counter-model for $A$ - that is to say, an MPT valuation $v$ such that $v(A)=0$. The existence of a winning strategy for $\mathbf{P}$ then corresponds to the impossibility to build such a countermodel and therefore to the validity of $A{ }^{13}$ After that, it is easy to apply the standard proof of completeness for MPT as it has been provided in Section 5 of Coniglio \& Silvestrini (2014).

The interpretation of the players' assertions concerning the three-valued matrices is actually straightforward. We first need to ensure that atomic formulas asserted in consistent sequences are not assigned the third value $\frac{1}{2}$. Outside consistent sequences, the translation is not much more complicated. Thus, in any play pertaining to a winning $\mathbf{P}$ strategy for $A$, atomic formulas are interpreted this way: (i) if $\perp_{a}$ is asserted by $\mathbf{O}$, then $a$ is assigned value $\frac{1}{2}$; (ii) otherwise, an atomic formula is assigned value 1 whenever it is asserted by $\mathbf{O}$, and atomic formulas not asserted by $\mathbf{O}$ receive value 0 . We notice that by definition (rule SR2 $2_{\mathbb{V}}$, it is not possible that $\perp_{a}$ be asserted if $a$ is asserted in a consistent sequence, which means that (i) is not possible in that case and our requirement is satisfied.

Now, by the rules of $D_{M P T}$ a victory by the Proponent always rests either on copying some of the Opponent's assertions of atomic formulas or by being allowed to introduce a non-explosive absurdity $\perp_{B}$ (for some formula $B$ ). From there it follows from the three-valued matrices for MPT and by induction on the complexity of the thesis $A$ that any valuation associated to the Opponent's atomic assertions as explained assigns to $A$ either of the designated values $\left\{1, \frac{1}{2}\right\}$. In other words: that if there is a winning $\mathbf{P}$ strategy in the $D_{M P T}$ game for $A$, then $A$ is MPT-valid.

After that our completeness theorem is obtained by applying Corollary 5.11 and Theorem 5.12 of Coniglio \& Silvestrini (2014).

\section{Conclusions and prospects}

The two dialogical systems we have introduced provide two quite different accounts of paraconsistency. In $D_{P 1}$ the game proceeds classically for the most part until one gets to the literals of the language. Paraconsistency is introduced and limited at the level of atomic formulas. The only modification made with respect to standard dialogical games is in the formal rule $\mathrm{SR} 2_{P 1}$, and it is made in such a way that it does not affect the other rules of the game. It is worth noticing that Sette's P1 has precisely this feature of having paraconsistency for atomic formulas but not for complex ones, and this explains why $D_{P 1}$ is an adequate dialogical approach to this logic. In $D_{M P T}$, paraconsistency is also introduced at the level of the formal rule, but in a much less restricted way. In

\footnotetext{
13 The reader can consult Sections 2.6.1 and 2.6.2 of Keiff(2007) for an example of this kind of demonstration in the case of classical logic.
} 
fact, the rules $\mathrm{SR} 2_{P 1}$ and $\mathrm{SR} 2_{\perp}$ of Section 2 , and the rule $\mathrm{SR} 2_{\mathbb{V}}$ of Section 3 , describe three different ways in which paraconsistency can "spread" within a system. As should be clear now, the rules we have studied in this work are not the only possible ones and it would be an interesting task to explore other possibilities and see how they relate with other paraconsistent logics, and in particular other LFIs.

Let us focus on the system $D_{M P T}$, which features a quite specific way to introduce and manage paraconsistency in a dialogical game. As we have said, paraconsistency is much less restricted than in $D_{P 1}$, in so far as it can spread within the game through negation and conjunction. The system is not without limits though, as paraconsistency is not transmitted through material implication. Moreover, and more importantly, $D_{M P T}$ features a way to block the transmission of paraconsistency by means of the operator $\mathbb{V}^{\circ}$ which opens consistent sequences for certain formulas in the course of the game. One way to understand it is that in $D_{M P T}$ the transmission of paraconsistency through negation and conjunction is not necessarily general: those formulas which are in the scope of $\mathbb{V}^{\circ}$ are protected from paraconsistency.

To this extent, our dialogical reconstruction of the paraconsistent logic MPT offers a formal framework which is applicable to situations in which inconsistent information may appear during certain argumentative interactions, but always within some limits and in particular in a way that there are some "safe" propositions for which inconsistency is not tolerated. ${ }^{14}$ This is captured in $D_{M P T}$ with how consistent sequences are opened by the operator $\mathbb{V}$, and how a formula once acknowledged as consistent stays consistent in the rest of a game, as we discussed in our explanation of $\mathrm{SR} 2 \mathbb{V}$.

The systems studied in this work as well as the preceding considerations open various possible directions for future work and we shall describe and explain some of them in what follows.

Further works on the operator $\circ$ and its dialogical treatment. One obvious direction is the study of other systems featuring the circle operator within the dialogical framework. In this quite general program, we can distinguish various specific directions. One is to explore to what extent a dialogical reconstruction of well-known LFIs can be proposed, and how. In this context, studying LFIs

\footnotetext{
14 It might be interesting to ask about the nature of the specific dialogues that logics like P1 and MPT would be modeling. When using paraconsistent logics, the idea is to start with games that are tolerant to inconsistencies. For example, if two agents discuss truth assignments to the arithmetic sentences or the naive set theory, in order to model this discussion we should tolerate the possibility that inconsistent truth assignments would arise because of the presence of the paradoxes. If we take as an example $\mathbf{P} \mathbf{1}$ and the circle-free fragment of MPT, such a thing will allow us to discuss such assignments without causing immediate triviality. But in the discussion between these two agents there will also be need for talking about the fragments of the "paradox-free" discussion, and for that we might want to recapture the classical contexts where contradictions would produce trivialization. Therefore, the idea is to extend the rules of the game with having punctual intolerance to contradiction for some formulas (the consistent ones, for which it will not be accepted that there is a contradiction). We want to thank an anonymous referee for calling us on to this point.
} 
which do not have truth tables (such as the basic logic mbC and some of its extensions) can result interesting because the dialogical framework would provide an alternative, game-theoretical, semantics for such logics. Moreover, instead of distinguishing between logics which have or do not have truth tables, another direction is to consider the difference between systems regarding rules of inference. In this work, we have limited our attention to two systems in which paraconsistency does not affect the material implication nor jeopardize Modus Ponens. Still, there are various paraconsistent systems in which this is not so, and the most well-known example is probably Asenjo-Priest's logic LP. Now, the circle operator $\circ$ can also be added to $\mathbf{L P}$ and allows to define a classical negation in the system, as well as possibly a second kind of implication with Modus Ponens. This suggests a path in which one can study paraconsistent dialogical systems without Modus Ponens and how reintroducing a certain amount of consistency in a dialogue can result in games with sequences in which Modus Ponens holds and others in which it does not.

Dialogues, paraconsistency and many-valued logics. It is worth insisting once again on the fact that the dialogical reconstructions we have provided in this work are not direct and plain multivalued dialogical games. The dialogical approach to meaning does not rest on the notion of truth and as such it is not a surprise that dialogical reconstructions of multivalued systems have received relatively few attention. The most notable exceptions are the works of Helge Rückert in 2004 and those of Fermüller and others as inspired by the approach of Giles(1979). By contrast, we have provided a dialogical reconstruction of two logics with three-valued matrices, P1 and MPT, without needing to implement a third value within our framework. On the contrary, paraconsistency is understood in the dialogical systems we have designed as a structural notion in the sense that it is introduced and managed at the structural level of the rules of the game that governs the use of absurdities $\perp_{X}$ by the players. Now, these general considerations open possible extensions of our work. One is related to the study of the relations between multivalued dialogical games and paraconsistent dialogical games, and in particular of the extent to where it is possible to provide a game-theoretical account of paraconsistency without needing to introduce the notion of multivaluation in the games. Are there LFIs or paraconsistent logics in general that cannot be accounted for in a dialogical setting without allowing multivaluation?

Another more general question stemming from the results of the current work is the following: are there other interesting ways to consider paraconsistency in dialogical games? Indeed we have seen how it can be introduced and controlled by means of the formal rule SR2. And it is quite clear that another way in which it can in principle be implemented is within many-valued dialogical games, but the dialogical framework (with its distinction between local or structural rules, and between play level and strategy level) may offer other fruitful ways to understand paraconsistency. Carnielli and Rahman (2000), for example, briefly considered a way to restrict explosion through the mechanism of repetition ranks that we have largely ignored in the current study. The most 
interesting aspect of this direction for future investigation is not merely to propose other equivalent ways to present the same logics again and again, but to delve into the various ways paraconsistency can occur in dialogue games of argumentation and, above all, if these differences are interesting in some kind of way.

In this line, it is important to take into account one recent direction presented in Beirlaen and Fontaine (2016). This work extends the approach of Rahman and Carnielli by implementing ideas and techniques from Inconsistency Adaptive Logic, in order to address some concerns expressed in van Bendegem (2001) and Rahman and van Bendegem (2002). This line of work is particularly interesting for us because, at first, it does seem to share some strong similarities with our work. Notably, it would be worth comparing the notion of reliable formulas they use with formulas called consistent in the context of LFIs, that is to say, formulas in the scope of a circle operator. Also, the system they study involves a change in the formal restriction similar to what we discussed in Section 3.1. In both cases, their approach also shows some differences, the main one being that the reliability of a formula and the change in the formal restriction are dealt with without making them explicit in the very language-object, as is the case with the circle and the dialogical $\mathbb{V}^{\circ}$ operators. Finally — and this is the topic of a more detailed analysis that goes beyond the scope of the present remarks - the approach developed by Beirlaen and Fontaine (2016) (because of the influence of Rahman and van Bendegem's work in 2002) seems to have important relation with the background of relevant logic, whereas this is not the case with our operator $\mathbb{V}^{\circ}$. This requires a more careful analysis and opens yet another interesting question. Can we combine our operator with techniques stemming from a relevantist and/or adaptive background?

Summing up, the dialogical framework offers new interesting perspectives in the study of paraconsistent systems in general. We believe that the results and reflections of this paper are the start of a new fruitful direction for research on Logic of Informal Inconsistency in particular and especially on the capacities and limits of the dialogical approach to this end.

Acknowledgements Eduardo Barrio is thankful to the Convenio de Desempeño Humanidades Artes y Ciencias Sociales (CDHACS) of University of Valparaíso for supporting and funding an academic visit in Valparaíso in 2016. Many thanks from Shahid Rahman to the Service Relations Internationales, the ADA Program of the MESHS and the UMR STL of University of Lille 3, as well as to the CDHACS of University of Valparaíso, for their support for the travels that made the meetings of the authors possible. Nicolas Clerbout is grateful to Conicyt and the CDHACS of the University of Valparaíso. The authors wish to thank Juan Redmond and Andrés Bobenrieth for fruitful discussions. Finally, we are especially thankful to anonymous referees for their helpful and very interesting comments and questions on a previous version of our work.

\section{References}

1. Beirlaen, M., and Fontaine, M., "Inconsistency-Adaptive Dialogical Logic", Logica Universalis 10(1), pp. 99-134 (2016) 
2. Carnielli, W., and Coniglio, M., Paraconsistent Logic: Consistency, Contradiction and Negation. Springer International Publishing (2016).

3. Carnielli, W., Coniglio, M., and Marcos, J., "Logics of Formal Inconsistency". In Gabbay, D. and Guenthner F. (eds.) Handbook of Philosophical Logic. Springer International Publishing, pp. 15-107 (2007).

4. Clerbout, N., "First-order dialogical games and tableaux". Journal of Philosophical Logic 43(4), pp. 785-801 (2014a)

5. Clerbout, N., "Finiteness of plays and the dialogical problem of decidability". IfCoLog Journal of Logics and their Applications 1(1), pp. 115-130 (2014b).

6. Clerbout, N., La Sémantique Dialogique. Notions fondamentales et éléments de metathéorie. College Publications, London (2014c).

7. Clerbout, N. and Rahman, S., Linking Game-Theoretical Approaches with Constructive Type Theory. Dialogical strategies, CTT demonstrations and the Axiom of Choice. Springer International PUblishing (2015).

8. Coniglio, M. and Silvestrini, L., "An alternative approach for quasi-truth". Logic Journal of the IGPL 22(2), pp. 387-410 (2014).

9. Da Costa, N., Sistemas Formais Inconsistentes. PhD thesis, Univesidade Federal do Paraná, Brazil, Curibita (1963).

10. Da Costa, N. "On the theory of inconsistent formal systems". Notre Dame Journal of Formal Logic 15(4), pp. 497-510 (1974).

11. Fermüller, C. G., "Dialogue games for many-valued logics: an overview". Studia Logica 90, pp. 43-68 (2008).

12. Giles, R., "A formal system for fuzzy reasoning". Fuzzy Sets and Systems 2, pp. 233-257 (1979).

13. Keiff, L., Le Pluralisme Dialogique. PhD thesis, Université Charles-de-Gaulle Lille 3, France (2007).

14. Rahman, S., Über Dialogue, Protologische Kategorien und andere Seltenheiten. Campus, New-York (1993).

15. Rahman, S. and Carnielli, W., "The dialogical approach to paraconsistency". Synthese 125, pp. 201-231 (2000).

16. Rahman, S. and Rückert, H., "Dialogical connexive logic". Synthese 127, pp. 105-139 (2001).

17. Rahman, S., and van Bendegem, J.-P., "The dialogical dynamics of adaptive paraconsistency". In Carnielli et al. (eds.) Paraconsistency: the logical way to the inconsistent, Marcel Dekker, pp. 295-322 (2002)

18. Rückert, H., "Logiques dialogiques multivalentes". Philosophia Scientiae 8(2), pp. 59-87 (2004).

19. Sette, A., "On the propositional calculus $P^{1}$ ". Mathematica Japonicae 18(13), pp. 173180 (1973)

20. Silverstrini, L., Uma Nova Abordagem Para A Noção De Quase-Verdade. PhD thesis, IFCH, State University of Campinas, Brazil (2011).

21. van Bendegem, J.-P., "Paraconsistency and dialogue logic: critical examination and further explorations". Synthese 127(1-2), pp. 35-55 (2001). 